\title{
Reconnection of Colliding Vortex Rings
}

\author{
Philippe Chatelain, Demosthenes Kivotides, and Anthony Leonard
}

Graduate Aeronautical Laboratories, California Institute of Technology, Pasadena, California 91125

(Received 3 September 2002; revised manuscript received 16 December 2002; published 6 February 2003)

We investigate numerically the Navier-Stokes dynamics of reconnecting vortex rings at small Reynolds number for a variety of configurations. We find that reconnections are dissipative due to the smoothing of vorticity gradients at reconnection kinks and to the formation of secondary structures of stretched antiparallel vorticity which transfer kinetic energy to small scales where it is subsequently dissipated efficiently. In addition, the relaxation of the reconnection kinks excites Kelvin waves which due to strong damping are of low wave number and affect directly only large scale properties of the flow.

In flow phenomena as diverse as quantum [1], magnetic [2], and incompressible [3] fluids, it is useful to study the physics of turbulence by modeling the system as a collection of tubular flux loops which in the case of vortical fields are called vortex filaments. An intrinsic property of such highly structured systems is their ability to dynamically change their topology via reconnection mechanisms. Does this change in topology affect in turn properties of fluid turbulence such as intermittency and scalar mixing (which depend directly on the structure of the flow) or the dynamics of energy in wave number space? Or is it the case that reconnection events are not generic and thus have no direct impact on the mean properties of turbulent flows? The aim of this Letter is to address these issues by fully resolving the NavierStokes dynamics of interacting vortex rings for three simple geometries having great potential for illuminating the physics of reconnection. Although the flows considered are not strictly turbulent, the hope is that in a future structural approach to the problem of turbulence a significant part of the flow complexity could be traced back to the physics of similar vortex interactions.

Incompressible vortex reconnections have an extensive bibliography (for a review of the work up to 1994, see $[4,5])$. In $[6,7]$ reconnections of vortex tubes were considered with an emphasis on the possibility of singularity formation as $\operatorname{Re} \rightarrow \infty$. In [8] the strong interactions between vortex rings were computed with the interest in developing numerical methods and turbulence models rather than in focusing on the physics of reconnection. In [9] it is discussed how a linked vortex configuration could be achieved starting from an unlinked initial state, and in [10] it is considered how the mixing of a nondiffusing passive scalar is affected during vortex ring collision. The reconnection of two approaching (but not colliding) vortex rings was studied experimentally in [11] and theoretically in [12]. This Letter extends these studies by considering generic vortex configurations and by capturing more features of vortex reconnections in a turbulent flow.
We solve the Navier-Stokes equations for an unbounded three-dimensional incompressible viscous flow. We employ the vorticity formulation:

$$
\begin{gathered}
\left(\frac{\partial}{\partial t}+\boldsymbol{u} \cdot \nabla\right) \boldsymbol{\omega}=(\nabla \boldsymbol{u}) \cdot \boldsymbol{\omega}+\nu \nabla^{2} \boldsymbol{\omega}, \\
\nabla \cdot \boldsymbol{u}=0,
\end{gathered}
$$

where $\boldsymbol{u}$ is the velocity and $\boldsymbol{\omega}$ is the vorticity. We use a vortex particle method [13]. In this method, the vorticity is discretized with Lagrangian elements. These elements which carry a vector-valued Gaussian distribution of vorticity are convected and stretched by the local velocity obtained by the Biot-Savart law. The complexity of the velocity computation is normally $\mathcal{O}\left(N^{2}\right)$ with $N$ being the number of particles; we have used a multipole algorithm that reduces this complexity to $\mathcal{O}(N \log (N))$. Viscous diffusion is handled by the particle strength exchange scheme.

We calculate the global kinetic energy $E$ and enstrophy $\Omega$ defined as

$$
\begin{aligned}
& E=\frac{1}{2} \int \boldsymbol{u} \cdot \boldsymbol{u} d \boldsymbol{x}, \\
& \Omega=\int \boldsymbol{\omega} \cdot \boldsymbol{\omega} d \boldsymbol{x} .
\end{aligned}
$$

For unbounded flows, the relation between kinetic energy and enstrophy is

$$
\frac{d}{d t} E=-\nu \Omega
$$

We also compute the evolution of the spectrum of the kinetic energy $E(k)$ which, in terms of the Fourier transform of vorticity $\hat{\boldsymbol{\omega}}=\left[1 /(2 \pi)^{3 / 2}\right] \int \boldsymbol{\omega}(\boldsymbol{r}) e^{-i \boldsymbol{r} \cdot \boldsymbol{k}} d \boldsymbol{r}$, is defined as

$$
E(k)=\frac{1}{2}(2 \pi)^{3} \int_{|k|=k} \hat{\boldsymbol{\omega}} \cdot \hat{\boldsymbol{\omega}}^{*} d \Omega_{k}
$$




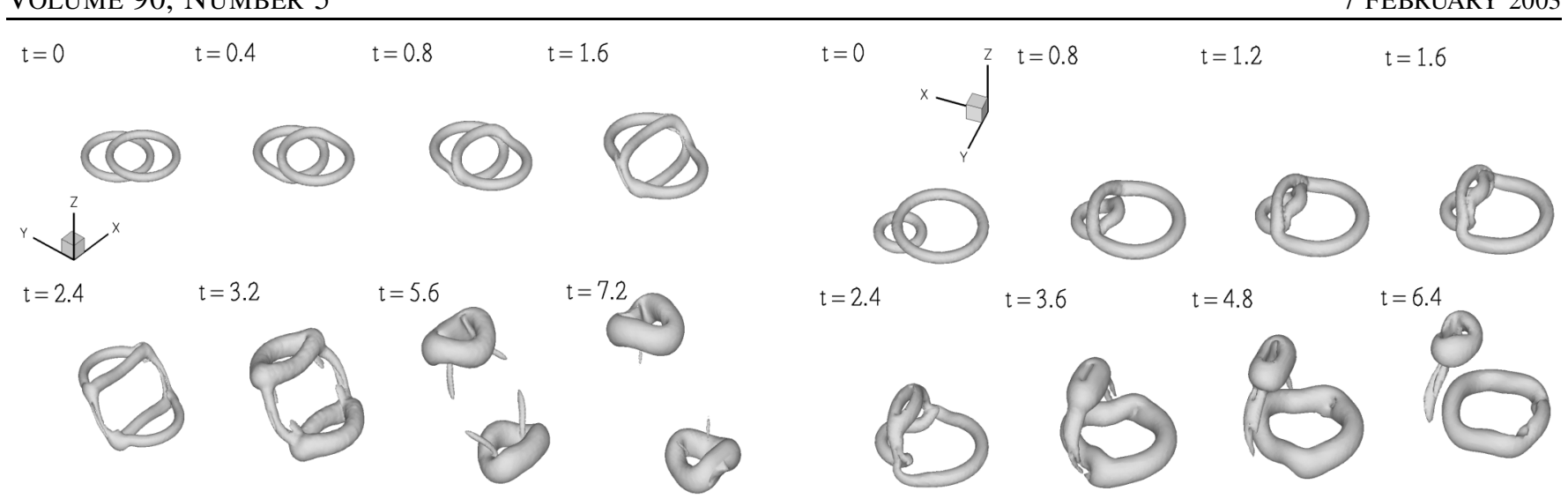

FIG. 1. Vortex rings in an offset collision: contours of vorticity; from $t=0$ to 2.4 , the contour is $\omega=0.15 \omega_{\max }^{t=0}$; for $t>2.4$, it is $\omega=0.025 \omega_{\max }^{t=0}$.

where $d \Omega_{k}$ denotes $\sin \theta_{k} d \theta_{k} d \phi_{k}$, the solid angle element in spherical coordinates. The calculation of the spectrum requires a double summation over the vortex elements which results to $\mathcal{O}\left(N^{2}\right)$ complexity. Because of this, the calculation of the spectrum is much more costly than the solution of the Biot-Savart law. Since the number of particles grows substantially during our simulations, from around $N=5 \times 10^{4}$ at $t=0$ to $8 \times 10^{5}$ in the end, our computational resources did not allow us to compute the spectra for all times.

All calculations were done with the same Reynolds number: $\operatorname{Re}=\frac{\Gamma}{\nu}=250$ where $\Gamma$ is the circulation of one ring and $\nu$ is the kinematic viscosity. This small value of the Re was dictated by the computational cost and the need for well-resolved reconnection regions. All the rings have the same initial $\Gamma$. All of our conclusions are conditioned upon the small value of the Reynolds number, as well as, on the common initial circulation and should not be extrapolated uncritically to other settings. The initial vorticity distribution in the cross section of every ring is Gaussian with a cutoff

$$
\omega_{\theta}=\frac{\Gamma}{2 \pi \sigma^{2}} e^{\left[\left(-r^{2}\right) /\left(2 \sigma^{2}\right)\right]},
$$

where $r$ is the distance to the core center, $\sigma$ is the core radius, and $\omega_{\theta}$ is the azimuthal vorticity. We chose $\sigma=$ $0.05 R$ (where $R$ is the radius of the ring), to ensure that the rings are still thin when reconnections occur. Our results were made dimensionless in the following manner: $t=$ $\left(\Gamma t^{\prime} / R^{2}\right), x=\left(x^{\prime} / R\right)$, and $\omega=\left[\left(R^{2} \omega^{\prime}\right) / \Gamma\right]$, where $t^{\prime}, x^{\prime}$, and $\omega^{\prime}$ are dimensional.

We studied three configurations. In the first case (Fig. 1), the initial rings are placed at a distance of $R / 4$ apart in the $z$ direction, offset by $R$ along the $y$ axis and they move in opposite directions along the $z$ axis. In the second case (Fig. 2), two rings of different radii ( $R$ and $R / 2$ ) and of initial separation $R / 4$ are moving in the same direction along the $z$ axis, with the center of the small
FIG. 2. Vortex rings of different radii: contours of vorticity; from $t=0$ to 2.4 , the contour is $\omega=0.15 \omega_{\max }^{t=0}$; for $t>2.4$, it is $\omega=0.05 \omega_{\max }^{t=0}$.

ring in a collision course with the circumference of the large one. The small ring has a larger self-induced velocity and catches up with the large ring. Finally, in the third case (Fig. 3), the two rings are linked at $90^{\circ}$ a ring going through the other in its center. One is moving in the positive $z$ direction; the other, in the positive $y$ direction.

All three evolutions lead to ring reconnection (Fig. 1-3) and have common features. The latter will be discussed here in the context of the first configuration observing that the phenomena are the same for the other two cases. The spectrum at $t=0$ (Fig. 4) has the characteristic oscillations of the spectrum of isolated vortex rings and a cutoff at the scale of ring core radius $\sigma=$ $0.05, k=20$. Our results (Fig. 1 and 5) suggest that (approximately) the reconnection starts around $t=0.6$ and ends around $t=1.75$ with a duration $\Delta t_{r}=1.15$. Specifically, as the rings approach each other, they stretch and deform near the collision points so that their respective vorticities become locally antiparallel. The two ends of this stretching region eventually become reconnection kinks in which (in the absence of singularities) the strong vorticity gradients are smoothed out by diffusion. This is

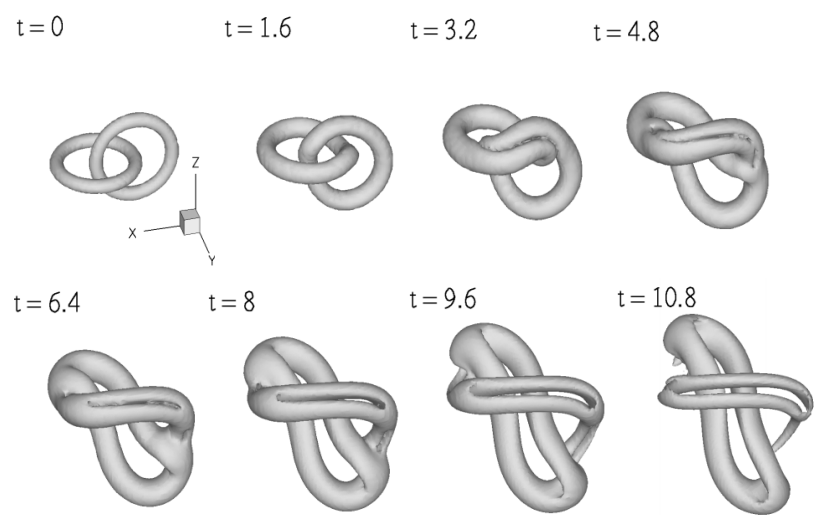

FIG. 3. Linked vortex rings: contours of vorticity; $\omega=$ $0.025 \omega_{\max }^{t=0}$. 


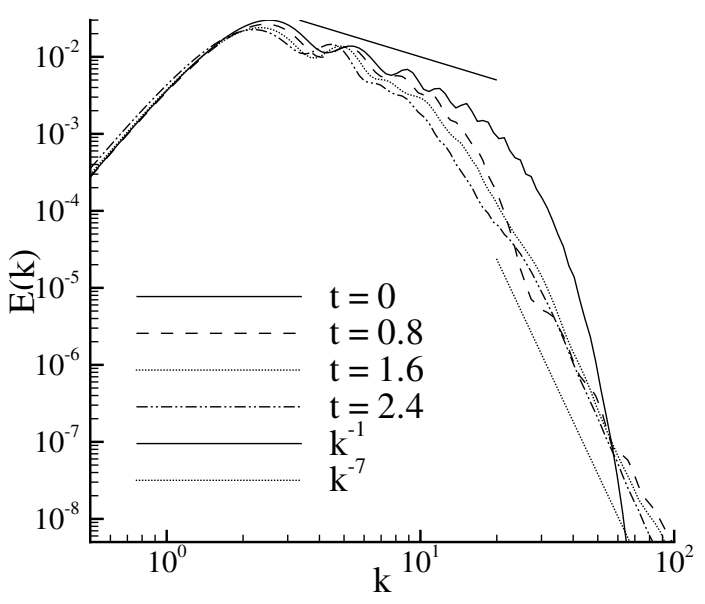

FIG. 4. Vortex rings in an offset collision: evolution of the energy spectrum.

also seen in the graphs of the global quantities (Fig. 5) where the beginning of the reconnection process corresponds to a hump in the graph of $\Omega$ and to a steepening of the slope of $E(t)$ between $t=0.6$ and $t=1.4$. Our calculation predicts that (due to diffusion) the filament core radius increases from the value $\sigma_{0}=0.05 R$ initially to the value $\sigma_{r}=0.12 R$ at the reconnection. Using this latter value we calculate the viscous time scale $t_{v}=$ $\sigma_{r}^{2} / \nu=0.36$. Scaling the convective ring velocity with $\Gamma / 4 \pi R$ we estimate the time needed for a ring to traverse $\sigma_{r}: t_{c}=4 \pi R \sigma_{r} / \Gamma=1.5$. These times are of the same order as $\Delta t_{r}$ and so it looks that both viscous and convective phenomena participate in the reconnection physics. The present relation between $t_{v}$ and $\Delta t_{r}$ is different from the one in [11] where the viscous scale was reported to be much larger than the duration of reconnection. However, in [11] the Reynolds number was 1600. The conclusion that the reconnection duration is inversely proportional to the Reynolds number and thus to the circulation of the vortices is plausible (also in agreement with $[6,14])$, but it is subject to the condition in [11] that the rings are merely touching themselves rather than colliding.

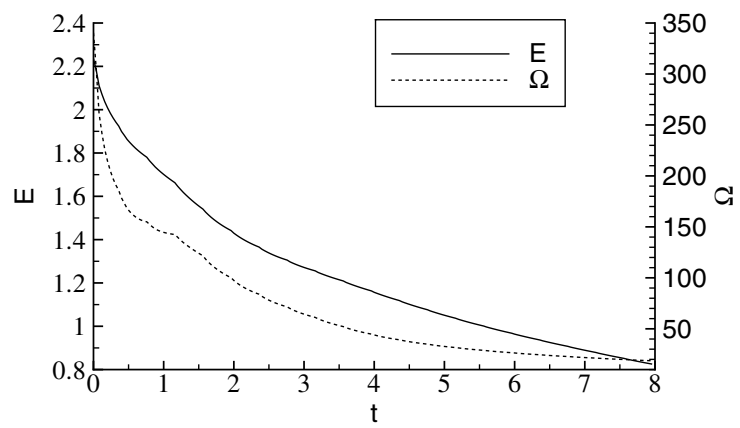

FIG. 5. Vortex rings in an offset collision: kinetic energy and enstrophy.

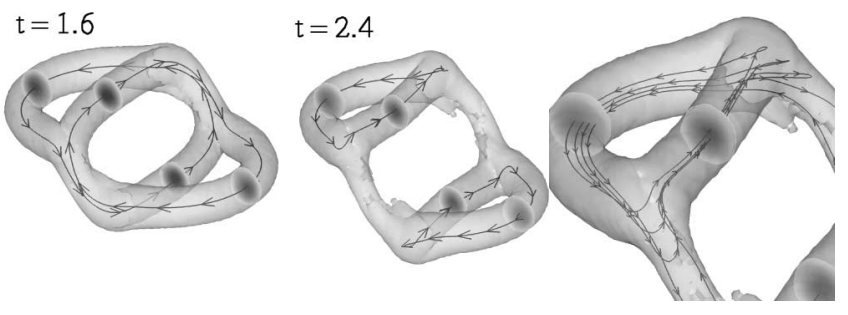

FIG. 6. Vortex rings in an offset collision: contour of vorticity and vortex lines at $t=1.6$ and 2.4; the transparent contour is $\omega=0.025 \omega_{\max }^{t=0}$; the vortex lines in (a) and (b) were chosen to pass through the vortex core center (the maximum value of $\omega$ ) at a location away from the reconnection kinks; in (c), a few neighboring lines are also shown for $t=2.4$.

After some time (Fig. 6), we can say that two new rings are formed. The pairs of filaments between the reconnection regions are stretched further as the new rings move apart from each other ( $t=1.6$ to 5.6). These stretched vorticity structures are responsible for a continued transfer of energy to the smallest scales until these structures are dissipated away. This conclusion is supported by the results of Fig. 4 where it is shown that the high wave number cutoff of the spectrum becomes a nonexponential one (although it remains still very steep) and that between $t=0.8$ and 2.4 there is a significant decay of the energy spectrum for $k<20$ but little change for $k>20$. This last observation indicates that in the small scales of motion an approximate balance between energy transfer from large scales due to stretching and local energy dissipation due to diffusion is attained (temporarily). This conclusion is also consistent with the vorticity structure shown in Fig. 7
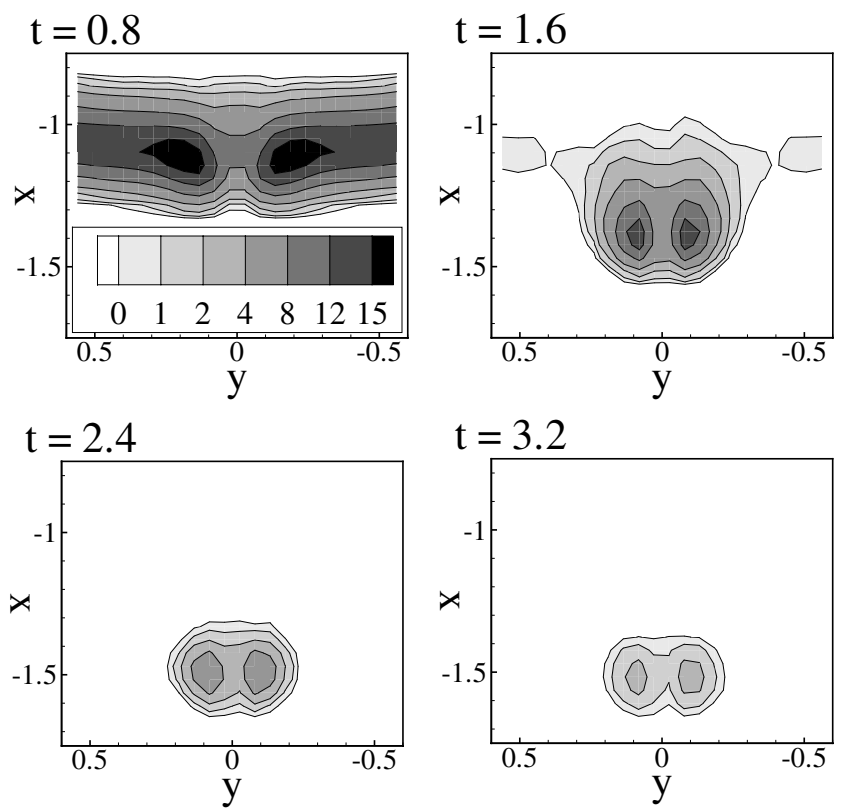

FIG. 7. Vortex rings in an offset collision: contours of vorticity magnitude in the plane $z=0$ from $t=0.8$ to 3.2. 
where it is observed that between $t=0.8$ and $t=2.4$ (the time of the last spectrum calculation), the vorticity magnitude in the secondary structures (where the global maximum of vorticity resides) stops increasing and in fact it decays slightly.

It is conceivable that for Reynolds numbers higher than 250 an intermediate scaling range (that is in between the $k^{-1}$ and $k^{-7}$ regimes) could appear with an inertial type of scaling. It is also expected that with increasing Reynolds number the hump observed in the global enstrophy during the reconnection could become more pronounced and (according to the previous discussion) shorter in duration.

In conclusion, we studied three generic vortex ring configurations and we found that in all cases the rings reconnect. This suggests that reconnection is a common phenomenon in vortex filament encounters and perhaps also in turbulent flows. In addition, we observe an intensification of dissipation which is local in time and could be a mechanism contributing to turbulence intermittency. A by-product of reconnection is the formation of stretched structures with antiparallel vorticity which transfer energy to the smallest scales where it is rapidly dissipated. Without this energy redistribution in wave number space the decay of global kinetic energy would have been slower. This important effect depends directly on the details of the initial vortex configuration (compare with experiments in [11]). The observed intensification of small scale motions hints to an enhancement of small scale mixing of passive scalars with $\mathrm{Sc} \geq 1$. The excited Kelvin waves represent a fast mechanism for energy transfer, but the small Reynolds number of our calculations is not suitable for understanding their full importance. In particular, they are confined to low wave numbers in opposition to the Kelvin waves observed in reconnections in quantum fluids [15]. This is because quantum filaments are inviscid and have a very thin core $(\sigma \sim 0.1 \mathrm{~nm})$ so that high wave number Kelvin waves propagate without damping even for rings with small circulation.
Besides illuminating important physics, the present work will guide future introduction of phenomenological reconnection models into vortex filament computational methods. In this way, the applicability of the latter methods will be extended to flows with complex vorticity configurations.

This research was partially supported by the Office of Naval Research and the Department of Energy.

[1] D. Kivotides, J.C. Vassilicos, D. C. Samuels, and C. F. Barenghi, Europhys. Lett. 57, 845 (2002).

[2] M. Christensson and M. Hindmarsh, Phys. Rev. D 60, 063001 (1999).

[3] A. Leonard, Annu. Rev. Fluid Mech. 17, 523 (1985).

[4] S. Kida and M. Takaoka, Annu. Rev. Fluid Mech. 26, 169 (1994).

[5] R. M. Kerr and F. Hussain, Physica (Amsterdam) 37D, 474 (1989).

[6] M. J. Shelley, D. I. Meiron, and S. A. Orszag, J. Fluid Mech. 246, 613 (1993).

[7] A. Pumir and R. M. Kerr, Phys. Rev. Lett. 58, 1636 (1987).

[8] G.S. Winckelmans, Annual Research Briefs, CTR Stanford, 1995.

[9] H. Aref and I. Zawadzki, Nature (London) 354, 50 (1991).

[10] I. Zawadzki and H. Aref, Phys. Fluids A 3, 1405 (1991).

[11] P. R. Schatzle, Ph.D. thesis, California Institute of Technology, 1987.

[12] W.T. Ashurst and D. I. Meiron, Phys. Rev. Lett. 58, 1632 (1987).

[13] G.-H. Cottet and P. Koumoutsakos, Vortex Methods, Theory and Practice (Cambridge University Press, Cambridge, 2000).

[14] J. F. Garten, J. Werne, D. C. Fritts, and S. Arendt, J. Fluid Mech. 426, 1 (2001).

[15] D. Kivotides, J. C. Vassilicos, D. C. Samuels, and C. F. Barenghi, Phys. Rev. Lett. 86, 3080 (2001). 